\title{
Formación docente y su relación con los contextos inclusivos.
}

(Teacher training and its relation to inclusive settings.)

\author{
Adrián Jesús Ricoy Cano \\ Universidad de Jaén (España)
}

Fecha recepción: 10-02-2018

Páginas 160-171

Fecha aceptación: 30-03-2018

\section{Resumo.}

El presente artículo trata sobre la formación de los docentes en neurociencias y cómo ese conocimiento es aprovechado para la creación de contextos inclusivos. Se muestra un estudio que abarca principalmente tres ámbitos: neuroeducación, formación docente y contextos inclusivos. El eje guía de esta investigación es la formación docente en neurociencias. El objetivo es analizar la formación de los docentes, haciendo hincapié sobre los conocimientos en neuroeducación y cómo ese conocimiento es aprovechado en la creación de contextos inclusivos. Para lograrlo se ha desarrollado un trabajo de investigación basado en una metodología cuantitativa. El instrumento de recogida de datos ha sido la escala de categorías.

Las conclusiones obtenidas en esta investigación manifiestan como la formación de los docentes en neurociencias y contextos inclusivos es escasa. Los resultados de esta investigación apuntan que los conocimientos de los docentes en neurociencias son insuficientes para relacionarlos con la creación de contextos inclusivos.

Palabras-clave: formación docente; neuroeducación; contextos inclusivos; aprendizaje; pedagogía

\section{Abstract.}

The following paper will discuss teaching training in neuroscience and how such training is used to create inclusive contexts. Such study comprises, mainly, three fields: neuroeducation, teaching training, and inclusive contexts. The main focus of this research is teaching training on neuroscience. The main goal of this paper is to analyze the training teachers get, closely considering their knowledge on neuroscience, and how such knowledge is used to create inclusive contexts. In order to achieve so, we have produced a research paper using a quantitative method. The data collection tool used on this paper is a categorical scale.

The conclusions from this investigation show how teaching training on neuroscience and inclusive contexts is rather is low. The results shown on these paper determine that teachers do not possess enough knowledge on neuroscience in order to create inclusive contexts.

Keywords: teacher training; neuroeducation; inclusive settings; learning; pedagogy 1.-Introducción. 
En la actualidad existen numerosas investigaciones acerca de los procesos de formación docente tanto en neurociencias, inclusión educativa, como en otros temas más genéricos. En relación a esto se puede destacar un estudio llevado a cabo por Triviño, Atuesta, Domínguez y Piñeres (2017). Para ellos el desafío actual es tratar de comprender qué aspectos se encuentran en desarrollo en los adolescentes y jóvenes universitarios, específicamente a nivel neurológico, para orientar desde allí las estrategias de acompañamiento, de manera que estén enfocadas al desarrollo de habilidades en los estudiantes y los avances alcanzados en estos espacios perduren en el desarrollo profesional del estudiante. Desde allí, la disciplina que mejor responde a este interés de articular el que hacer docente con las Neurociencias, es la neuroeducación.

En cuanto a la formación docente en inclusión para Correa, Sierra y Alzate (2014) las posibilidades de enfoques para la atención a la diversidad son múltiples, su elección debe ser coherente con las características de los contextos educativos; el enfoque de derechos es el más promocionado en las dos últimas décadas para garantizar el derecho a la educación, pues en éste la inclusión es una necesidad de desarrollo social y responde a una problemática polifacética. La educación inclusiva no es sinónimo de cobertura o de acceso a un servicio educativo y debe superar la referencia de los colectivos en espacios y circunstancias en los que se señala a los grupos por su situación de vulnerabilidad y no por su condición humana. Resignificar la educación en el enfoque de inclusión implica una mirada en dos sentidos. De un lado, de quien es el excluido y discriminado y de otro, ajustar las condiciones de contexto para hacer efectivo el derecho a la educación. La inclusión no es atribución exclusiva de las instituciones educativas, es un compromiso ético, una responsabilidad política y una condición necesaria para la viabilidad y la gobernabilidad en marcos de tolerancia.

A lo largo de este artículo se hablará de neuroeducación. La neuroeducación trata de usar los conocimientos basados en la neuroimagen y pretende lanzar tentativas que atiendan al modo de interactuar del cerebro con su entorno en cada proceso de enseñanza-aprendizaje. (Béjar, 2014). Se reflexionará también sobre los procesos de formación docente y sobre contextos inclusivos como ya se adelantaba al inicio de este epígrafe. En este sentido Ortiz (2015) llevó a cabo una investigación sobre mejoras en los estudiantes que asistían a escuelas inclusivas. Los resultados confirman un hallazgo consensual en la literatura, a saber, que la mixtura social de las escuelas es favorable para el rendimiento de los estudiantes de bajo NSE que asisten a ellas. Para terminar, se tratará de relacionar todos estos términos a fin de valorar cuantitativamente si la formación de los docentes en neurociencias influye en su capacidad para crear espacios inclusivos.

\section{2.-Formación docente en inclusión.}

En este epígrafe se vincularán los conceptos de formación docente e inclusión. Se comenzará viendo qué significa formación docente en diversidad y qué criterios se siguen para la formación en educación inclusiva. También se argumentará por qué el 
liderazgo inclusivo es una propuesta potente para la creación de contextos inclusivos en la escuela actual, y se mostrará cómo es necesaria una preparación específica para poder llevarlo a cabo. Por último, se pasará a observar los resultados obtenidos en una investigación sobre la formación/preparación docente en atención a la diversidad.

Se empezará este apartado con una aproximación a la noción de formación docente en inclusión. Para ello, se seguirá a Durán y Giné (2017), quienes argumentan que se puede definir la educación inclusiva, como un proceso de formación, en un sentido amplio; un proceso de capacitación de los sistemas educativos, de los centros y del profesorado para atender la diversidad del alumnado. Se trata pues de un verdadero reto de formación del profesorado, no como tarea individual, sino como un proceso de desarrollo profesional y de mejora de los centros y los sistemas educativos. La formación del profesorado para la diversidad será útil para desarrollar una educación de mayor calidad para todos si se configura como un aspecto del sistema educativo que ayuda al cambio de la cultura profesional docente (reconstrucción de sus procesos de identidad y desarrollo profesional), en un contexto abierto a todos y orientado por valores inclusivos. No se trata pues de una formación individual para el desarrollo profesional aislado, sino más bien de una capacitación personal para participar de una actividad docente que permita el desarrollo profesional del profesorado y la mejora del centro. (Durán y Giné, 2017)

En este sentido, la formación deberá ir orientada a la creación de un profesional que reflexiona sobre su práctica, en el seno de una organización educativa; que colabora activamente para mejorar su competencia y la del centro; y que actúa como un intelectual crítico y consciente de las dimensiones éticas de su profesión (Durán y Giné, 2017) (como ya aparecía citado en Arnaiz, 2003).

Resulta de interés conocer cuáles son los criterios generales de fundamentación de la formación para la educación inclusiva, estos pueden ser concretados siguiendo los aportados por Echeita (2006), y que podrían sintetizarse según Durán y Giné (2017) en:

- Pedagogía de la complejidad: los problemas educativos tienen una dimensión múltiple (psicológica, social, moral); una resolución incierta y están sometidos a conflictos de valores, imprevisibles y simultáneos.

- Perspectivas constructivistas: el aprendizaje de los alumnos en situación de vulnerabilidad no es cualitativamente distinto al de los demás. Las aportaciones de la concepción constructivista, como el triángulo interactivo, la construcción del conocimiento o los mecanismos de influencia educativa, son instrumentos útiles.

- Desarrollo integrado: el desarrollo del alumnado debe basarse en aportaciones como las inteligencias múltiples, especialmente la interpersonal e intrapersonal, así como el sentimiento de competencia, construido sobre la autoestima y los patrones atribucionales ajustados. 
- Enseñanza adaptativa: la gestión inclusiva del aula requiere la definición de objetivos básicos para todos, con distinto nivel de consecución, y la diversificación de actividades y grados de ayudas.

- Adaptaciones curriculares: la toma de decisiones de cambios en la acción educativa debe promover la participación de los implicados y basarse en una evaluación psicopedagógica contextual, intentando ser lo menos restrictiva posible y valorándose periódicamente.

- Red de apoyos y colaboraciones: las aulas y los centros deben promover las ayudas y el trabajo cooperativo entre alumnos, profesores, familias y comunidad.

- Escuelas como centros de la mejora: el proceso de avance de las escuelas debe de ser planificado, llevado a la práctica a través de acciones que puedan ser evaluadas y replanteadas en ciclos de mejora.

- Diversidad como fuente de enriquecimiento y de estímulo para la innovación: es necesario adoptar una actitud que permita ver la diversidad como un mecanismo que, generando incertidumbre y desafío, crea condiciones para la excelencia.

Cambiando un poco el enfoque, pero sin dejar de lado la idea de formación docente e inclusión. Se puede percibir cómo existe un creciente interés en el estudio del liderazgo escolar por su repercusión en la práctica y en la política de los sistemas y centros educativos inclusivos. Según destacan Macmillan y Edmunds (2010), hay evidencias significativas de que el éxito de los programas de inclusión es atribuible, en gran parte, al liderazgo ejercido por el director y a su capacidad para crear una escuela inclusiva. (León, Crisol y Arrebola, 2018)

Este tipo de liderazgo está contextualizado, se muestra comprometido con la diferencia (Gunter, 2006) y es crítico con las formas de escolarización homogéneas que se han mostrado inapropiadas para alcanzar una educación de calidad para todos y todas, sobre todo para aquellas personas más vulnerable. (León, Crisol y Arrebola, 2018)

Para León, et al. (2018), citado en Angelides, Antoniu y Chalambous (2010) el liderazgo inclusivo ejerce una influencia positiva en la práctica del profesorado, en la calidad de los aprendizajes y participación de los estudiantes (Ainscow y Kaplan, 2005), así como en la mejora de los centros y en la creación una cultura inclusiva. Las tareas y/o funciones desempeñadas por el equipo directivo para cumplir las metas de un centro inclusivo han sido agrupadas por nosotros en estudios anteriores (León, 2001, 2012; León et al., 2016) en: i) Apertura a la comunidad, que implica llevar a cabo iniciativas desde el centro educativo que potencian la apertura del centro hacia al entorno y la familia; ii) El Centro como comunidad inclusiva, para lo que el equipo directivo emprende acciones para generar una visión compartida, promoviendo la participación, cooperación y dinámicas de reflexión positiva de la 
comunidad educativa hacia la diversidad; iii); Gestión de los procesos de enseñanzaaprendizaje, llevando a cabo iniciativas para mejorar y favorecer la coordinación en el proceso de enseñanza y aprendizaje del profesorado y iv) Comunidad Profesional de Aprendizaje, el líder inclusivo promueve la formación, el desarrollo profesional del profesorado y la creación de comunidades profesionales de aprendizaje (León, Crisol y Arrebola, 2018). Esta última función es la que desde este artículo se destacaría, para que se aprecie cómo esta propuesta del líder inclusivo relaciona formación docente e inclusión, pues la puesta en funcionamiento de este programa lleva implícitos procesos de formación en inclusión para los docentes.

Sin embargo, después de haber visto cómo es la formación docente en inclusión, surge la duda de si realmente los egresados por las Universidades, que han obtenido el título de educación primaria o básica, están capacitados para atender al alumnado con dificultades. Una reciente investigación llevada a cabo en la Universidad Técnica de Machala ofrece unos resultados muy interesantes que se señalan a continuación.

Al preguntarles a los graduados de la Universidad Técnica de Machala si consideran estar preparados didáctica y metodológicamente para atender a estudiantes con necesidades educativas especiales, de acuerdo a los lineamientos del Ministerio de Educación, en consenso respondieron que "muy poco". Opinan que necesitan apropiarse de recursos didácticos y desarrollar competencias y capacidades que les permitan el acompañamiento del aprendizaje de estos estudiantes. (Izquierdo, Cuenca, Cuenca, 2018)

Los entrevistados estiman que "los docentes de la educación regular no tienen la formación didáctica y metodológica necesarias para atender a diversidad". En tal sentido Sánchez, Díaz, Sanhueza y Fernández (2008) y Sánchez (2011) en sus averiguaciones determinaron que los estudiantes de pedagogía consideran que los docentes de la educación regular no están preparados para desarrollar una adecuada atención a los alumnos con esas necesidades diversas.

Similares resultados obtuvieron en sus investigaciones Álvarez (2002); Marchesi \& Martin (2002); Jiménez (2003) y Fernández (2013), los que concluyeron que el docente percibe estar bien preparado en los contenidos de la materia que imparte, pero no es así para atender la diversidad; Idol (2006) agrega que según sus observaciones muchos docentes no se sienten competentes ni a gusto enseñando a niños con diversas necesidades postura que evidencia la pobre disposición anímica para enfrentar una labor de alto contenido humano. (Izquierdo, Cuenca, Cuenca, 2018)

Se ha podido ver a lo largo de toda esta sección que pese a la buena fundamentación que tiene la formación docente en atención a la diversidad, y a pesar de propuestas tan novedosas como la del líder inclusivo, dicha formación sigue siendo insuficiente e insatisfactoria en la mayoría de los casos, como así se muestra en los resultados obtenidos en la investigación llevada a cabo por Izquierdo, Cuenca, Cuenca, (2018). 


\section{3.-Neuroeducación: formación docente en neurociencias.}

Sobre el origen de la neuroeducación Campos (2014) afirma que, durante los últimos veinte cinco años, las investigaciones en el campo de la neurociencia han provocado un fuerte impacto en la educación y con ello, surgieron muchas preguntas acerca de cómo unir investigación y práctica, de tal forma que esto implique un avance en los sistemas educativos. En la actualidad, se comienza a identificar las formas en que se podrán unir aprendizaje y cerebro para producir la mejora de la calidad de la educación y del perfil del educador. Entre todos los desafíos que plantearon las investigaciones neurocientíficas, estaban aquellos relacionados a la comprensión de los procesos cognitivos, de las bases biológicas de la cognición, del lenguaje, del aprendizaje y memoria, de las emociones 0 de las funciones ejecutivas, todos innegablemente vinculados al campo de la educación. Los resultados que fueron emergiendo de estas áreas de investigación, en muy poco tiempo, no solo despertaron la curiosidad de los educadores, sino que han provocado un movimiento a nivel mundial para que se articularan los conocimientos neurocientíficos con el mundo educativo.

En este sentido, viene emergiendo la Neuroeducación como una nueva línea de pensamiento y acción que tiene como principal objetivo acercar a los agentes educativos a los conocimientos relacionados con el cerebro y el aprendizaje, considerando la unión entre la Pedagogía, la Psicología Cognitiva y las Neurociencias. Para Campos (2010) este acercamiento entre educadores y neurocientíficos ha empezado a dar grandes frutos, comenzando por romper algunos "neuromitos" que fueron masivamente esparcidos cuando las Neurociencias empezaron a llegar a los ámbitos educativos.

En necesario pues, la formación y capacitación de los educadores en Neuroeducación para que comprendan los mecanismos cerebrales que subyacen al aprendizaje, a la memoria, al lenguaje, a los sistemas sensoriales y motores, a la atención, a las emociones y todo lo que el medio puede influir en ello. Campos (2010)

\section{4.-Relación entre inclusión y neuroeducación.}

Ya se sabe, que la neuroeducación abre nuevas fronteras en el ámbito de la pedagogía. Uno de estos cauces lleva a meditar sobre la utilidad que tiene la neurodidáctica en relación a la inclusión educativa. Recientemente, como forma de conexión entre estos dos conceptos, aparece la noción de "Neurodiversidad". No son muchas las definiciones existentes en la actualidad en referente a esta terminología debido a su reciente aparición.

Entre las más destacadas se puede denotar la de Masataka (2017), el cual habla de Neurodiversidad, y con ella se refiere a la noción de que las propiedades cognitivas y emocionales aparentemente "alteradas" características de los trastornos del desarrollo como los trastornos del espectro autista (TEA) no son necesariamente déficits, sino que entran en las variaciones normales de comportamiento exhibidas 
por los humanos o "una idea que afirma atípica (neurodivergente) el desarrollo neurológico es una diferencia humana normal que debe ser reconocida y respetada como cualquier otra variación humana ". El término fue acuñado por primera vez a fines de la década de 1990 por personas que participaban en movimientos por los derechos civiles, y ha contribuido a ayudar a las personas con necesidades especiales a vivir su vida social. En particular, ha cobrado impulso en la comunidad de TEA y se está extendiendo más allá para incluir grupos con otras categorías de trastornos del desarrollo, incluidos problemas de aprendizaje, discapacidades intelectuales y el trastorno por déficit de atención / hiperactividad (TDAH) porque insta a discutir la diversidad cerebral usando el mismo tipo de discurso que se emplea al hablar de biodiversidad y diversidad cultural.

Las implicaciones de la neurodiversidad han sido más notables para la educación. La noción ha cambiado con éxito la visión de la intervención con esas personas, desde el intento de superar lo que es su debilidad hasta el de aumentar su fuerza: se ha producido un cambio de paradigma. En lugar de ubicar a los niños en categorías de discapacidad separadas y utilizar herramientas y lenguaje obsoletos para trabajar con ellos, los maestros que participan en educación especial y regular pueden usar herramientas y lenguaje inspirados por el movimiento de ecología para diversificar el aprendizaje y ayudar a los niños a triunfar en el aula. (Masataka, 2017)

\section{5.-Diseño de la investigación.}

De acuerdo con los objetivos planteados se intenta delimitar el método de estudio desde la investigación en educación. En palabras de Pérez Ferra (2009) el método es el procedimiento para alcanzar los objetivos y la metodología constituye el estudio del método. Para ello, se toma como referencia la clasificación aportada por Bisquerra (1989) el cual plantea que los métodos de investigación pueden estar orientados 0 bien a obtener conocimiento básico, o bien a obtener conocimiento aplicado a la toma de decisiones y a las acciones para el cambio. Esta investigación es de naturaleza exploratoria y descriptiva, se adoptará una metodología cuantitativa, pues los propósitos son apreciar mediante resultados numéricos cómo es la formación de los docentes en Educación Primaria, tanto en aspectos generales, como más concretamente en conocimientos sobre neuroeducación. Otra de las pretensiones es valorar si los maestros/as hacen uso de las neurociencias en sus prácticas pedagógicas con el objetivo de crear contextos más inclusivos. Por último, se pretende valorar qué saben docentes sobre inclusión y si son capaces de diferenciar esta terminología de otras. Para todo esto una metodología cuantitativa se hace imprescindible, tanto para valorar los resultados obtenidos como para hacer comparaciones entre los mismos.

\section{1.-Problema.}

El problema que se destaca en esta investigación sería el siguiente:

La formación de los docentes de educación primaria en neurociencia ¿influye en la creación de los contextos inclusivos? 


\section{2.-Objetivos generales y específicos.}

Se distinguen dos tipos de objetivos en esta investigación: generales y específicos. Estos se muestran a continuación.

\section{General}

Analizar la formación de los docentes de educación primaria, haciendo hincapié sobre los conocimientos de los mismos en neuroeducación y cómo ese conocimiento es aprovechado en la creación de contextos inclusivos.

\section{Específicos}

1- Advertir el uso que hacen los docentes de sus conocimientos en neuroeducación en la escuela.

2- Analizar si los docentes se preocupan en sus prácticas diarias por la creación de contextos inclusivos.

3- Estimar si los maestros aprecian la diferencia entre los conceptos de integración e inclusión escolar.

4- Indagar sobre la percepción de los maestros de Educación Primaria en lo referente a su formación inicial y continua como docentes, así como la adecuación de la misma.

5- Valorar el conocimiento de los maestros sobre el concepto de inclusión.

6- Determinar el nivel de conocimiento sobre neuroeducación de los docentes de primaria.

7-Revisar aspectos relacionados con la formación docente en neuroeducación.

\section{3.-Población y muestra.}

La población susceptible de ser investigada la forman docentes de Educación Primaria de los centros docentes de una provincia andaluza, independientemente de cuál sea su especialidad. El total de maestros y maestras de esta provincia es de 3446, de acuerdo con la última actualización de Consejería de educación (2018). La muestra está constituida por 346 maestros y maestras. Los educadores seleccionados para el muestreo se encuentran en activo e imparten docencia en Educación Primaria. La técnica de muestreo probabilístico utilizada para la selección de la muestra ha sido el aleatorio simple. Otzen y Manterola (2017) dicen que este método garantiza que todos los individuos que componen la población blanco tienen la misma oportunidad de ser incluidos en la muestra. 


\section{4.-Instrumentos.}

Para la realización de este estudio, se opta por la utilización de una escala como instrumento de recogida de datos.

Con toda probabilidad, la escala de categorías constituye una de las técnicas de medida de creencias, preferencias y actitudes más utilizada por los científicos de la conducta. En palabras de Dawes (1975), se trata de "la omnipresente escala de clasificación" (pág. 115). Son muchos los términos asociados a la escala de categorías: escala de clasificación, escala de juicio absoluto, escala cerrada, escala de valoración resumida, escala de múltiple elección, escala tipo Likert, etc. En cualquier caso, bajo todas estas denominaciones se hace referencia a un procedimiento de escalamiento en el que el sujeto asigna los estímulos a un conjunto específico de categorías o cuantificadores lingüísticos, en su mayoría, de frecuencia (siempre, a veces, nunca, etc.,) o de cantidad (todo, algo, nada, etc.). Cañadas y Sánchez (1998)

\section{5.-Análisis de resultados.}

El análisis de resultados de esta investigación se llevó a cabo mediante un proceso de análisis estadístico. Se valora cada ítem de la escala con un pequeño comentario con el que se pretende destacar dichos resultados. Véase el siguiente ejemplo:

Gráfico A1.- Como docente he recibido conocimientos a lo largo de mis estudios sobre neuroeducación.

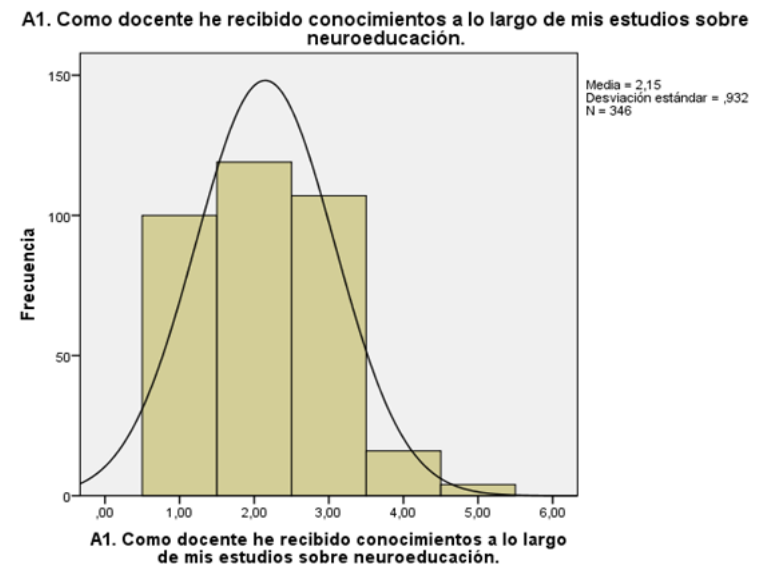

Fuente: elaboración Propia

En este primer ítem se preguntó a los encuestados si habían recibido formación a lo largo de las carreras académicas sobre neuroeducación. En esta gráfica se puede apreciar el valor de la media aritmética, que es el valor característico de una serie de datos cuantitativos. En este caso la $\bar{x}=2,15$. La mayor concentración de los valores se encontraría entorno al 2,15. El tipo de curtosis Leptocúrtica, el coeficiente de 
curtosis (en adelante g2) es mayor que cero, g2>0. En estas encuestas aparece una escala en la cual se debe de seleccionar su nivel de conocimiento con respecto a lo que se pregunta (donde 1 es nada, 2 es poco, 3 es algo, 4 es bastante y 5 es mucho). La desviación típica marca la situación del 95\% de los datos. De forma que si se restase a la media aritmética la desviación típica y se señalara el punto resultante y por otro lado se sumara a la misma media nuevamente la desviación típica y se marcase dicho punto, entre tales puntos se encontraría el 95\% de los datos como se mencionaba anteriormente.

Se puede concluir abstrayendo, que en la mayoría de los casos se ha recibido poca formación sobre neuroeducación a lo largo de los estudios. Algo que se cree es muy grave para la pedagogía actual y sobre lo que se debería de poner medidas para su mejora en un futuro próximo.

Este es un ejemplo del total de 30 ítems que componen la escala y los cuales fueron analizados minuciosamente uno por uno.

\section{6.-Conclusiones.}

Las principales conclusiones obtenidas en esta investigación muestran como la formación de los docentes de educación primaria en neurociencias es poca, a pesar de su actitud positiva a continuar formándose en esta área. En cuanto a conocimientos específicos en neurociencias y contextos inclusivos, los resultados alertan que estos son escasos, sin embargo, en el aula los docentes tratan de llevar a cabo técnicas basadas en los mismos, aunque no tienen una base científica que los sustente. Los resultados presentados en este estudio concluyen que los conocimientos demostrados por los docentes en neurociencias son insuficientes para relacionarlos con la creación de contextos inclusivos.

\section{6.-Referencias.}

Béjar, M. (2014). Una mirada sobre la educación. Neuroeducación. Padres y maestros, $\quad 355, \quad 49-52$ Recuperado de http://revistas.upcomillas.es/index.php/padresyma estros/article/view/2622/232

Bisquerra Alzina, R. (1989). Introducción conceptual al análisis multivariable. Un enfoque informático con los paquetes SPSS-X, BMDP, LISREL y SPAD. Barcelona: PPU.

Campos, A. L. (2010). Neuroeducación: uniendo las neurociencias y la educación en la búsqueda del desarrollo humano. La educ@ción revista digital, 143, 1-14. Recuperado de http://kdoce.cl/wp-content/uploads/2017/10/DOC1-neuroeduc acion.pdf

Campos, A. L. (2014). La Neuroeducación: descartando neuromitos y construyendo principios sólidos. Centro Iberoamericano de Neurociencias, Educación y 
Desarrollo Humano, SN, 1-15. Recuperado de http://www.cerebrum.la/congre somundial/papers/contenido/Anna\%20Lucia/Neuroeducacion-ALC.pdf

Cañadas Osinski, I. y Sánchez Bruno, A. (1998). Categorías de respuesta en escalas tipo Likert. Psicotema Revista Anual de Psicología, 10, (3), 623-631. Recuperado de file:///C:/Users/PcCom/Desktop/7489-13192-1-PB.pdf

Correa, J., Bedoya Sierra, M., Agudelo Alzate, G. (2014). Formación de docentes participantes en el programa de educación inclusiva con calidad en Colombia. Interior Revista Latinoamericana educación inclusiva, 9 (1), 43-61. Recuperado de https://dialnet.unirioja.es/servlet/articulo?codigo=5155476

Durán Gisbert, D. y Giné Giné C. (2017). La formación del profesorado para la educación inclusiva: Un proceso de desarrollo profesional y de mejora de los centros para atender la diversidad. Red Iberoamericana De Expertos En La Convención De Los Derechos De Las Personas Con Discapacidad. Recuperado de http://repositoriocdpd.net:8080/bitstream /handle/123456789/1 913/ArtDuranGisbertDFormaciondelprofesorado.pdf?sequence=1

León, M. J, Crisol, E., y Moreno Arrebola R. (2018). Las Tareas del Líder Inclusivo en Centros Educativos de Zonas Desfavorecidas y Favorecidas. Revista Iberoamericana sobre Calidad, Eficacia y Cambio en Educación, 16, (2), 2140. Recuperado de https://revistas.uam. es/index.php/reice/article/view/9282

Masataka, N. (2017). Implications of the idea of neurodiversity for understanding the origins of developmental disorders. Physics of Life Reviews, 20, 85-108. DOI: $\underline{10.1016 / j . p l r e v ~ .2 ~ 016.11 .002 ~}$

Ortiz, I. (2015). Escuelas inclusivas en el contexto de segregación social del sistema escolar chileno. Calidad en la educación, (42), 93-122. Recuperado de https://scielo.conicyt.cl/pdf/caledu/n42/art04.pdf

Otzen, T. y Manterola, C. (2017). Técnicas de muestreo sobre una población a estudio. International Journal of Morphology, 35 (1): 227-232. Recuperado de https://scielo.conicyt.cl/pdf/iimorphol/v35n1/art37.pdf

Pérez Ferra M. 2009. La Europa del conocimiento, un referente axiológico para la Universidad, en A. Medina, M. Sevillano, M. L. y Torre, S. de la (Coordinadores). Una universidad para el siglo XXI. Espacio Europeo de Enseñanza Superior. Madrid: Universitas.

Tinoco-Izquierdo, W.E, Tinoco-Cuenca, N.P. y Carchi Cuenca, C. (2018). Una aproximación cualitativa a la educación inclusiva en el contexto ecuatoriano. Maestro y Sociedad. Revista electrónica para Maestros y profesores, 1 (15). Recuperado de http://revistas.uo.edu.cu/index.php/MyS/article/view/3295/290 $\underline{9}$ 
Revista Internacional de Apoyo a la Inclusión, Logopedia, Sociedad y Multiculturalidad.

Volumen 4, Número 2, Abril 2018, ISSN: 2387-0907, Dep. Legal: J-67-2015

http://riai.jimdo.com/

Triviño Riaño, A., Cel y Atuesta, D., Triana Domínguez, C. y Gutiérrez de Piñeres, C. (2017). Neuroeducación: una revisión teórica con miras al fortalecimiento de la permanencia estudiantil en contextos universitarios. Congresos Clabes. Recuperado de http://www.revistas.utp.ac.pa/index.php/clabes/article/view/16 $\underline{28 / 2365}$ 